Research Article

\title{
An Efficient Teaching Model of International Cooperation Based on Artificial Intelligence
}

\author{
Meng Xiao' ${ }^{1}$ and Haibo Yi $\mathbb{i D}^{2}$ \\ ${ }^{1}$ School of Management, Shenzhen Polytechnic, Shenzhen 518055, China \\ ${ }^{2}$ School of Artificial Intelligence, Shenzhen Polytechnic, Shenzhen 518055, China \\ Correspondence should be addressed to Haibo Yi; haiboyi@szpt.edu.cn
}

Received 28 July 2021; Revised 12 September 2021; Accepted 21 September 2021; Published 30 September 2021

Academic Editor: Punit Gupta

Copyright (c) 2021 Meng Xiao and Haibo Yi. This is an open access article distributed under the Creative Commons Attribution License, which permits unrestricted use, distribution, and reproduction in any medium, provided the original work is properly cited.

\begin{abstract}
International cooperation in running schools has promoted the scale of higher education and trained a number of talents with international vision. However, there are many differences between domestic teaching and foreign teaching in the international cooperation in running schools. This makes it difficult for students to adapt to the teaching of foreign universities. Connecting domestic teaching with foreign teaching and solving the problem of differentiation has become an important issue in international cooperation in running schools. In order to improve the quality of international cooperation of high education, it is very urgent to design an efficient model of international cooperation. We exploit artificial intelligence to design a teaching model of international cooperation. We implement the effective model of international cooperation in logistics management and software technology, where the implementation results show that the model can provide learning suggestions of the foreign stage according to the learning situation of the domestic stage.
\end{abstract}

\section{Introduction}

In recent years, international cooperation in running schools has become a popular mode of higher education [1]. It is a common mode of international cooperation to study in domestic universities for 1-3 years and finish their studies in foreign universities in the remaining time.

In China, almost all universities have international cooperation programs. In the undergraduate colleges, " $2+2$ " cooperative mode is usually adopted, where students complete the first two years of study in China and the second two years in foreign countries and obtain the diploma of foreign universities or domestic and foreign universities. In higher vocational colleges, " $3+1$ " cooperative mode is usually adopted, where students complete the first three years of study in China and the last year in foreign countries and obtain a diploma from domestic universities and bachelor's degree from foreign universities.

International cooperation in running schools has promoted the scale of higher education and trained a number of talents with international vision. International cooperation in running schools has been widely concerned and accepted by many students and parents. Compared with direct study abroad, parents and students have lower learning costs in international cooperative education and can experience different types of higher education at home and abroad at the same time. International cooperation in running schools is very beneficial to the cultivation of interdisciplinary talents.

However, in the international cooperation in running schools, there are many differences between domestic teaching and foreign teaching. This makes it difficult for students to adapt to the teaching of foreign universities. Connecting domestic teaching with foreign teaching and solving the problem of differentiation has become an important issue in international cooperation in running schools.

In order to improve the quality of international cooperation of high education, it is very urgent to design an efficient model of international cooperation. Artificial intelligence technology is a very popular information 
technology in recent years, and the application of artificial intelligence in the field of education is gradually emerging [2-6]. Chiu and Chai proposed sustainable curriculum planning for artificial intelligence education [7]. Calvo et al. proposed a multidisciplinary PBL approach for teaching industrial informatics and robotics in engineering [8]. Marques et al. enhanced the student learning experience in software engineering project courses [9]. Aktan et al. applied distance learning to control engineering laboratories [10]. Graesser et al. proposed an intelligent tutoring system with mixed-initiative dialogue [11]. Hoic-Bozic et al. proposed a blended learning approach to course design and implementation [12]. The strong point of artificial intelligence technology is that it can model and analyze and predict according to the past data [13-20].

We exploit artificial intelligence to design a teaching model of international cooperation. Taking logistics management and software technology as examples, we analyze the learning situation of domestic students based on artificial intelligence and establish the training set of artificial intelligence model; then, based on artificial intelligence, we analyze the learning situation of foreign students and establish the labels of artificial intelligence model; finally, through artificial intelligence training, we build an effective model of international cooperation.

We implement the effective model of international cooperation in logistics management and software technology, where the implementation results show that the model can provide learning suggestions of the foreign stage according to the learning situation of the domestic stage. Compared with other models, the artificial intelligence-based model is more intelligent.

We present the rest of content in the following organization. Section 2 presents an overview of artificial intelligence. Section 3 proposes an efficient teaching model of international cooperation. Section 4 presents the results of implementation and comparison. Section 5 presents the conclusion of this paper.

\section{Preliminaries}

Artificial intelligence technology is a very popular information technology in recent years, and the application of artificial intelligence in the field of education is gradually emerging. The strong point of artificial intelligence technology is that it can model and analyze and predict according to the past data.

One of the main techniques of artificial intelligence is deep learning. Deep learning plays an important role in medical diagnosis and analysis. Klang proposed deep learning and medical imaging [21]. Haskins et al. proposed deep learning in medical image registration [22]. Ba proposed medical sports rehabilitation deep learning system of sports injury based on MRI image analysis [23]. $\mathrm{Xu}$ proposed a deep learning method to predict lung cancer from serial medical imaging [24]. Sourati et al. proposed intelligent labeling based on fisher information for medical image segmentation using deep learning [25].
In addition, deep learning also plays an important role in agriculture, industry, commerce, and other fields. Liu et al. proposed a new fluid factor and its application using a deep learning approach [26]. Hu et al. proposed deep collaborative learning with application to multimodal brain development study [27]. Asanuma et al. proposed deep learning applications to topology optimization of electric motor [28]. Al-Sharman et al. proposed deep learning-based neural network training for state estimation enhancement and application to attitude estimation [29]. Zhang et al. proposed deep learning-based mobile application isomorphic GUI identification for automated robotic testing [30]. Trivizakis et al. proposed extending 2D convolutional neural networks to 3D for advancing deep learning cancer classification with application to MRI liver tumor differentiation [31]. Yan et al. proposed soft sensor modeling method based on semisupervised deep learning and its application to wastewater treatment plant [32].

\section{An Efficient Teaching Model of International Cooperation}

3.1. Overview of the Scheme. In order to improve the quality of international cooperation of high education, it is very urgent to design an efficient model of international cooperation. Artificial intelligence technology is a very popular information technology in recent years, and the application of artificial intelligence in the field of education is gradually emerging. The strong point of artificial intelligence technology is that it can model and analyze and predict according to the past data.

We exploit artificial intelligence to design a teaching model of international cooperation based on artificial intelligence. In higher vocational colleges, " $3+1$ " cooperative mode is usually adopted, where students complete the first three years of study in China and the last year in foreign countries and obtain a diploma from domestic universities and bachelor's degree from foreign universities.

We take " $3+1$ " program of logistics management and software technology as examples.

(1) We analyze the learning situation of domestic students based on artificial intelligence and establish the training set of the artificial intelligence model, which is illustrated in Section 3.2.

(2) Based on artificial intelligence, we analyze the learning situation of foreign students and establish the labels of artificial intelligence model, which is illustrated in Section 3.3.

(3) Through artificial intelligence training, we build an effective model of international cooperation, which is illustrated in Section 3.4.

3.2. Collecting Training Data. We take " $3+1$ " program of logistics management and software technology in higher vocational colleges as examples.

(1) We analyze the learning situation of domestic students based on artificial intelligence. 
" $3+1$ " cooperative mode is usually adopted in higher vocational colleges, where students complete the first three years of study in China and the last year in foreign countries and obtain a diploma from domestic universities and bachelor's degree from foreign universities.

Core courses of logistics management and software technology in higher vocational colleges include Python, Java, introduction to computer, computer network, data structure and introduction to logistics, logistics planning and design, procurement and supply management, procurement project management, and transportation management, which is summarized in Table 1 .

(2) Based on the learning situation of domestic students, we establish the training set of artificial intelligence model.

We establish the training set based on the scores of the core courses of logistics management and software technology in higher vocational colleges. First, we convert all points that are not a percentage system to a hundred mark system. Second, we limit the range of data from 0 to 1 by dividing all fractions by 100 . We illustrate the process of establishing training set in Table 2 .

3.3. Labeling Training Data. Based on artificial intelligence, we analyze the learning situation of foreign students and establish the labels of artificial intelligence model.

In " $3+1$ " cooperative mode, students complete the last year in foreign countries and obtain a diploma from domestic universities and bachelor's degree from foreign universities.

Courses of logistics management in foreign countries include warehouse management, distribution management, international logistics, international trade theory and practice, procurement process exercise, transportation technique, warehousing management practice, logistics distribution center design, international logistics practice, success study, innovation, quality development training, etc.

Courses of software technology in foreign countries include calculation method, compiling principle, software engineering, operating system principle, database system principle, network database, microcomputer principle and assembly language programming, web-based programming, software development technology, software testing technology, multimedia technology, network security technology, etc.

We label the training set based on the scores of the core courses of logistics management and software technology in the colleges of foreign countries. First, we convert all points that are not a percentage system to a hundred mark system. Second, we limit the range of data from 0 to 1 by dividing all fractions by 100 . We illustrate the process of labeling training set in Table 3 .

3.4. Building Model. Through artificial intelligence training, we build an effective model of international cooperation, which is depicted in Figure 1.
(1) Collecting training data and label: " $3+1$ " cooperative mode is usually adopted in higher vocational colleges, where students complete the first three years of study in China and the last year in foreign countries and obtain a diploma from domestic universities and bachelor's degree from foreign universities. We take " $3+1$ " program of logistics management and software technology as examples. We collect scores of core courses of logistics management and software technology in higher vocational colleges and foreign colleges as training data and labels.

(2) Selecting features of the model: we select ten core courses for logistics management and software technology in higher vocational colleges as the features, respectively. We use $l_{1}, l_{2}, \ldots, l_{10}$ to denote ten core courses for logistics management in higher vocational colleges and use $s_{1}, s_{2}, \ldots, s_{10}$ to denote ten core courses for software technology in higher vocational colleges.

(3) Designing model: we select ten core courses for logistics management and software technology in foreign colleges as the labels, respectively. We use $m_{1}, m_{2}, \ldots, m_{10}$ to denote ten core courses for logistics management in foreign colleges and use $t_{1}, t_{2}, \ldots, t_{10}$ to denote ten core courses for software technology in foreign colleges. Then, we need to find the relation between the training set and labels, i.e., training set $l_{1}, l_{2}, \ldots, l_{10}, s_{1}, s_{2}, \ldots, s_{10}$ and labels $m_{1}, m_{2}, \ldots, m_{10}, t_{1}, t_{2}, \ldots, t_{10}$.

We build an efficient model based on artificial intelligence. We build a five-layer architecture with the inputs of training set and the outputs of the labels. We take logistics management as an example and depict the architecture in Figure 2.

(4) Training model with the training data and label: we summarize the five-layer architecture in Table 4 . The input layer represents the scores of the ten core courses for logistics management or software technology in higher vocational colleges. The output layer represents the scores of the ten core courses for logistics management or software technology in foreign colleges. Besides, we design three hidden layers, where each layer includes 100 nodes with full connections. In order to find the relation between inputs and outputs, we use the training set and label to train the model.

\section{Result}

4.1. Implementation. We implement the effective model of international cooperation in logistics management and software technology, where we use Python programming language with the version 3.6 and TensorFlow deep learning tool.

(1) We collect data based on " $3+1$ " cooperative mode, which is usually adopted in higher 
TABLE 1: Core courses of logistics management and software technology.

\begin{tabular}{lcc}
\hline & Software technology & Logistics management \\
\hline First year & Python, introduction to computer & Introduction to logistics \\
Second year & Java, computer network & Procurement project management \\
Third year & Data structure & Transportation management \\
\hline
\end{tabular}

TABLE 2: Establishing training set.

\begin{tabular}{lccc}
\hline & Original score & First stage & Second stage \\
\hline Python & $\mathrm{A}+$ & 100 & 1 \\
C++ & $\mathrm{A}$ & 95 & 0.95 \\
Introduction to computer & $\mathrm{B}$ & 85 & 0.85 \\
Introduction to logistics & $\mathrm{C}$ & 75 & 0.75 \\
Java & $\mathrm{D}$ & 65 & 0.65 \\
Computer network & $\mathrm{F}$ & 0 & 0 \\
Procurement project management & 100 & 100 & 1 \\
Data structure & 60 & 60 & 0.6 \\
Transportation management & 0 & 0 & 0 \\
\hline
\end{tabular}

TABLE 3: Labeling training set.

\begin{tabular}{lccc}
\hline & Original score & First stage & Second stage \\
\hline Warehouse management & $\mathrm{A}+$ & 100 & 1 \\
International logistics practice & $\mathrm{A}$ & 95 & 0.95 \\
Distribution management & $\mathrm{B}$ & 85 & 0.85 \\
International logistics & $\mathrm{C}$ & 75 & 0.75 \\
Success study & $\mathrm{D}$ & 0 & 0.65 \\
Compiling principle & $\mathrm{F}$ & 100 & 0 \\
Software engineering & 100 & 60 & 1 \\
Operating system principle & 60 & 0 & 0.6 \\
Database system principle & 0 & & 0 \\
\hline
\end{tabular}

TABLE 4: Five-layer architecture.

\begin{tabular}{lccc}
\hline & Type & Nodes & Number of nodes \\
\hline First layer & Input & $l_{1}, l_{2}, \ldots, l_{10}$ & 10 \\
Second layer & Hidden & $h_{1,1}, l_{1,2}, \ldots, l_{1,100}$ & 100 \\
Third layer & Hidden & $h_{2,1}, l_{2,2}, \ldots, l_{2,100}$ & 100 \\
Fourth layer & Hidden & $h_{3,1}, l_{3,2}, \ldots, l_{3,100}$ & 100 \\
Fifth layer & Output & $t_{1}, t_{2}, \ldots, t_{10}$ & 10 \\
\hline
\end{tabular}

vocational colleges. Students complete the first three years of study in China and the last year in foreign countries and obtain a diploma from domestic universities and bachelor's degree from foreign universities. We take " $3+1$ ” program of logistics management and software technology as examples. We collect scores of ten core courses of logistics management and software technology in higher vocational colleges and foreign colleges as training data and labels.

(2) We convert all points that are not a percentage system to a hundred mark system. Then, we limit the range of data from 0 to 1 by dividing all fractions by 100.
(3) We build a five-layer architecture with the inputs of training set and the outputs of the labels.

(4) We collect testing data to test the architecture.

We summarize the implementation details in Table 5, where the accuracy of the model is $91.6 \%$.

(1) A training set of 2000 samples is used in training the model.

(2) A testing set of 500 samples is used in testing the model.

(3) Inputs are $l_{1}, l_{2}, \ldots, l_{10}, s_{1}, s_{2}, \ldots, s_{10}$.

(4) Outputs are $m_{1}, m_{2}, \ldots, m_{10}, t_{1}, t_{2}, \ldots, t_{10}$.

The implementation results show that the model can provide learning suggestions of the foreign stage according to the learning situation of the domestic stage.

4.2. Comparison. We compared the traditional teaching mode with the AI-based teaching mode from four aspects: data-driven, subjectivity/objectivity, technology, and intelligence. The comparison results are summarized in Table 6. Compared with other models, the artificial intelligence-based model is more intelligent. 


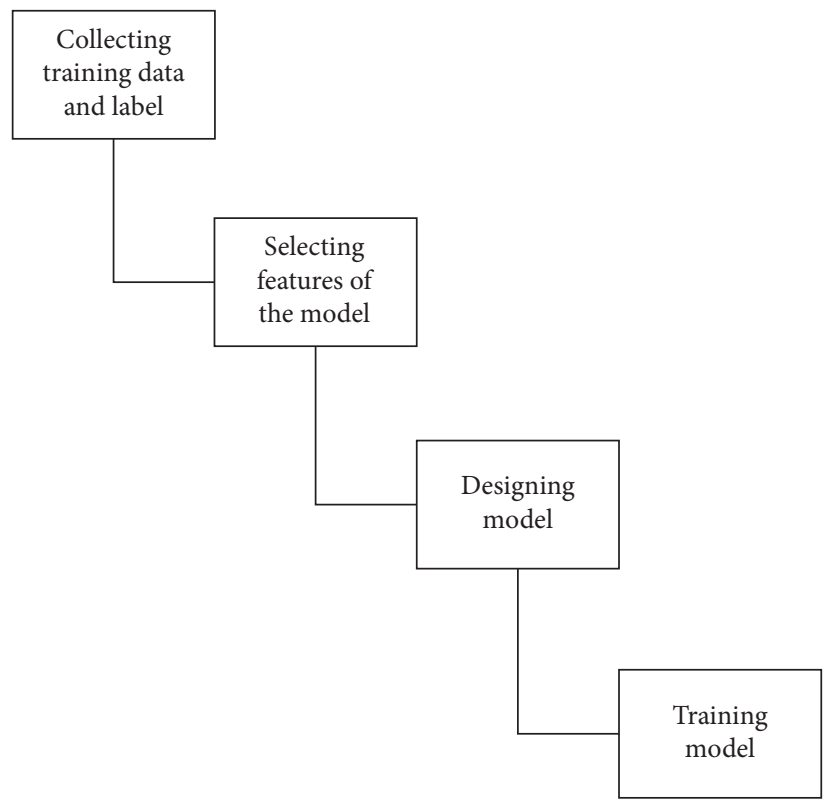

FIgURE 1: Building model.

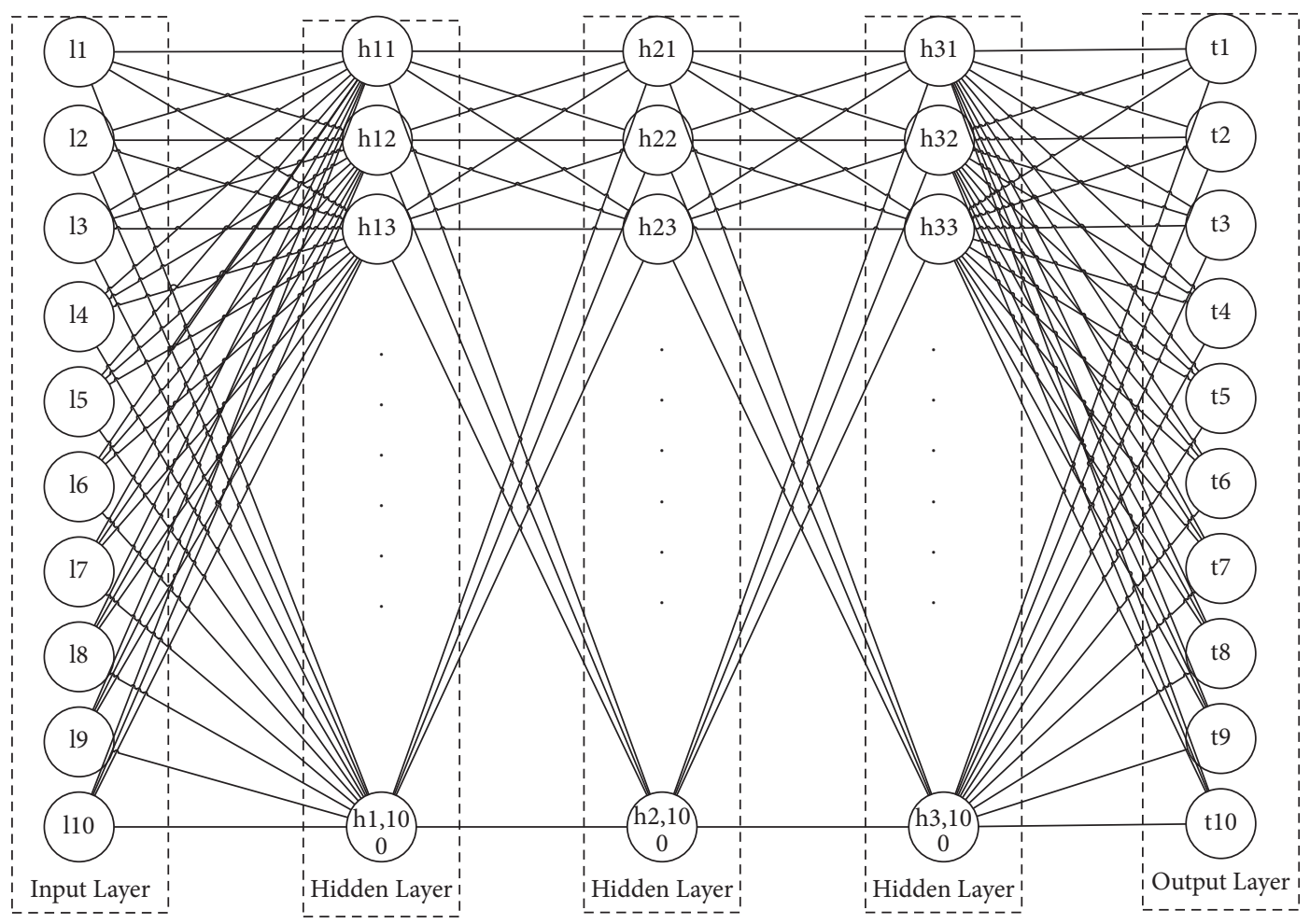

FIgURE 2: Five-layer architecture. 
TABLE 5: Implementation results.

\begin{tabular}{lcccc}
\hline Training set & Testing set & Inputs & Outputs & Accuracy \\
\hline 2000 & 500 & 20 & 20 & 91.6 \\
\hline
\end{tabular}

TABle 6: Comparison results.

\begin{tabular}{lcccc}
\hline & Data-driven & Subjectivity/objectivity & Technology used & Model intelligence \\
\hline Traditional teaching model & No & Subjectivity & Information technology & Low \\
AI teaching model & Yes & Objectivity & Artificial intelligence & High \\
\hline
\end{tabular}

\section{Conclusions}

In the international cooperation in running schools, there are many differences between domestic teaching and foreign teaching. In order to improve the quality of international cooperation of high education, it is very urgent to design an efficient model of international cooperation. Artificial intelligence technology is a very popular information technology in recent years, and the application of artificial intelligence in the field of education is gradually emerging. The strong point of artificial intelligence technology is that it can model and analyze and predict according to the past data. We exploit artificial intelligence to design a teaching model of international cooperation based on artificial intelligence. Taking logistics management and software technology as examples, we analyze the learning situation of domestic students based on artificial intelligence and establish the training set of artificial intelligence model; then, based on artificial intelligence, we analyze the learning situation of foreign students and establish the labels of artificial intelligence model; finally, through artificial intelligence training, we build an effective model of international cooperation.

We implement the effective model of international cooperation in logistics management and software technology, where the implementation results show that the model can provide learning suggestions of the foreign stage according to the learning situation of the domestic stage. Compared with other models, the artificial intelligence-based model is more intelligent.

\section{Data Availability}

No data were used to support this study.

\section{Conflicts of Interest}

The authors declare that they have no conflicts of interest.

\section{Acknowledgments}

The authors acknowledge Guangdong Province 2021 Educational Science Planning Project (no. DSYJ108), Shenzhen Science and Technology Program (grant no. 20200821082500001), and Teaching Research and Practice Project of Shenzhen Polytechnic (no. 7019310019J).

\section{References}

[1] L. Sisi, L. Baocun, and J. E. Aoun, "Robot-proof: higher education in the age of artificial intelligence," Higher Education, vol. 1, pp. 1-3, 2018.

[2] L. Deng, "Artificial intelligence in the rising wave of deep learning: the historical path and future outlook [perspectives]," IEEE Signal Processing Magazine, vol. 35, no. 1, pp. 180-177, 2018.

[3] Q. Zhang, L. T. Yang, Z. Chen, and P. Li, "A survey on deep learning for big data," Information Fusion, vol. 42, pp. 146-157, 2018.

[4] X. Sun, P. Wu, and S. C. H. Hoi, "Face detection using deep learning: an improved faster RCNN approach," Neurocomputing, vol. 299, no. 19, pp. 42-50, 2018.

[5] H. Chen, O. Engkvist, Y. Wang, M. Olivecrona, and T. Blaschke, "The rise of deep learning in drug discovery," Drug Discovery Today, vol. 23, no. 6, pp. 1241-1250, 2018.

[6] Q. S. Zhang and S. C. Zhu, "Visual interpretability for deep learning: a survey," Frontiers of Information Technology \& Electronic Engineering, vol. 19, no. 1, pp. 27-39, 2018.

[7] T. K. F. Chiu and C.-s. Chai, "Sustainable curriculum planning for artificial intelligence education: a self-determination theory perspective," Sustainability, vol. 12, no. 14, p. 5568, 2020.

[8] I. Calvo, I. Cabanes, J. Quesada, and Q. Barambones, "A multidisciplinary PBL approach for teaching industrial informatics and robotics in engineering," IEEE Transactions on Education, vol. 61, pp. 1-8, 2018.

[9] M. Marques, S. F. Ochoa, M. C. Bastarrica, and F. J. Gutierrez, "Enhancing the student learning experience in software engineering project courses," IEEE Transactions on Education, vol. 61, pp. 1-11, 2018.

[10] B. Aktan, C. A. Bohus, L. A. Crowl, and M. H. Shor, "Distance learning applied to control engineering laboratories," IEEE Transactions on Education, vol. 39, no. 3, pp. 320-326, 2002.

[11] A. C. Graesser, P. Chipman, B. C. Haynes, and A. Olney, "AutoTutor: an intelligent tutoring system with mixed-initiative dialogue," IEEE Transactions on Education, vol. 48, no. 4, pp. 612-618, 2005.

[12] N. Hoic-Bozic, V. Mornar, and I. Boticki, "A blended learning approach to course design and implementation," IEEE Transactions on Education, vol. 52, no. 1, pp. 19-30, 2009.

[13] L. Li, Y. L. Lin, N. N. Zheng et al., "Artificial intelligence test: a case study of Intelligent vehicles," Artificial Intelligence Review, vol. 50, 2018.

[14] D. S. W. M. P. Dai, "Human intelligence needs artificial intelligence," Sensors, vol. 5855, no. 3, pp. 95-99, 2018.

[15] J. He, S. L. Baxter, J. Xu, J. Xu, X. Zhou, and K. Zhang, “The practical implementation of artificial intelligence technologies in medicine," Nature Medicine, vol. 25, 2019. 
[16] C. Qi, A. Fourie, Q. Chen, and Q. Zhang, "A strength prediction model using artificial intelligence for recycling waste tailings as cemented paste backfill," Journal of Cleaner Production, vol. 183, no. 10, pp. 566-578, 2018.

[17] Y. Varatharajah, B. Berry, J. Cimbalnik et al., "Integrating artificial intelligence with real-time intracranial EEG monitoring to automate interictal identification of seizure onset zones in focal epilepsy," Journal of Neural Engineering, vol. 15, 2018.

[18] W. Pan and P. Suping, "A new scheme to improve the performance of artificial intelligence techniques for estimating total organic carbon from well logs," Energies, vol. 11, no. 4, p. 747, 2018.

[19] X. Zhang, Y. Wang, C. Liu, and Z. Chen, "A novel approach of battery pack state of health estimation using artificial intelligence optimization algorithm," Journal of Power Sources, vol. 376, no. feb.1, pp. 191-199, 2018.

[20] Y. Takefuji, "In the long run, the gap between the rich and poor will dramatically increase by artificial intelligence," Science, vol. 1, 2018.

[21] E. Klang, "Deep learning and medical imaging," Journal of Thoracic Disease, vol. 10, no. 3, pp. 1325-1328, 2018.

[22] G. Haskins, U. Kruger, and P. Yan, "Deep learning in medical image registration: a survey," Machine Vision and Applications, vol. 31 , no. $1-2,2020$

[23] H. Ba, "Medical sports rehabilitation deep learning system of sports injury based on MRI image analysis," Journal of Medical Imaging and Health Informatics, vol. 10, no. 5, pp. 1091-1097, 2020.

[24] Y. Xu, A. Hosny, R. Zeleznik et al., "Deep learning predicts lung cancer treatment response from serial medical imaging," Clinical Cancer Research, vol. 25, 2019.

[25] J. Sourati, A. Gholipour, J. G. Dy, X. Tomas-Fernandez, S. Kurugol, and S. K. Warfield, "Intelligent labeling based on Fisher information for medical image segmentation using deep learning," IEEE Transactions on Medical Imaging, vol. 38, no. 11, pp. 2642-2653, 2019.

[26] C. Liu, D. P. Ghosh, A. M. A. Salim, and W. S. Chow, "A new fluid factor and its application using a deep learning approach," Geophysical Prospecting, vol. 67, no. 1, pp. 140-149, 2019.

[27] W. Hu, B. Cai, A. Zhang, V. Calhoun, and Y.-P. Wang, "Deep collaborative learning with application to multimodal brain development study," IEEE Transactions on Biomedical Engineering, vol. 66, p. 1, 2019.

[28] J. Asanuma, S. Doi, and H. Igarashi, "Transfer learning through deep learning: application to topology optimization of electric motor," IEEE Transactions on Magnetics, vol. 56, no. 99, p. 1, 2020.

[29] M. K. Al-Sharman, Y. Zweiri, M. A. K. Jaradat, R. Al-Husari, and D. Gan, "Deep-learning-based neural network training for state estimation enhancement: application to attitude estimation," IEEE Transactions on Instrumentation and Measurement, vol. 69, pp. 1-11, 2019.

[30] T. Zhang, Y. Liu, J. Gao, L. P. Gao, and J. Cheng, "Deep learning-based mobile application isomorphic gui identification for automated robotic testing," IEEE Software, vol. 37, 2020 .

[31] E. Trivizakis, G. C. Manikis, K. Nikiforaki et al., "Extending $2 \mathrm{D}$ convolutional neural networks to $3 \mathrm{D}$ for advancing deep learning cancer classification with application to MRI liver tumor differentiation," IEEE Journal of Biomedical and Health Informatics, vol. 23, p. 1, 2018.
[32] W. Yan, R. Xu, K. Wang, T. Di, and Z. Jiang, "Soft sensor modeling method based on semisupervised deep learning and its application to wastewater treatment plant," Industrial \& Engineering Chemistry Research, vol. 59, no. 10, pp. 4589-4601, 2020. 